\title{
Magnetic and Thermal Performance of a Conduction Cooled Splittable Quadrupole
}

R. Carcagno, A. Hemmati, H. Hayano, V. S. Kashikhin, M.J. Kim, N. Kimura, L. Kokoska, S. Kotelnikov, J. Nogiec, D. F. Orris, R. Pilipenko, C. Sylvester, M. Takahashi, M. A. Tartaglia, T. Taizo, T. Wokas, A. Yamamoto

\begin{abstract}
A superconducting quadrupole magnet with splittable yoke has been designed for use in ILC Main Linac (ML) cryomodules. The splittable yoke allows assembly around the beam pipe, to avoid potential contamination of the superconducting RF cavities. The magnet is cooled by conduction and covers the full range of required ML field gradients. A critical requirement is stability of the magnetic center, at the level of 5 microns, for a $20 \%$ variation of the operating field. We report here the results of thermal, quench, and magnetic performance tests of a prototype splittable quadrupole, that were made up to the maximum design operating gradient in a conduction-cooled test cryostat.
\end{abstract}

Index Terms-Superconducting Quadrupole, Conduction Cooled, Cryomodule Focusing Element, Prototype, Magnetic Center, Quench.

\section{INTRODUCTION}

$\mathrm{T}$ HE INTEREST in developing quadrupole focusing elements for linear accelerator superconducting cryo-modules remains strong within the international community. A prototype ILC cryomodule is presently being assembled at $\mathrm{KEK}$, and it will incorporate a new prototype conductioncooled superconducting quadrupole based upon the most recent Fermilab splittable quadrupole design that was developed [1-2] to meet specifications for the ILC [3]. A parallel development effort is ongoing at Fermilab, of a splittable quadrupole doublet magnet to be used in cryomodules for the ASTA facility [4].

The splittable quadrupole design allows the magnet to be prepared and tested independently of other cryomodule components, and assembled around the beam tube, which eliminates concern about contaminating the ultra-clean superconducting RF environment. This approach naturally leads to a design with indirect coil cooling by conduction, rather than needing a complicated helium vessel.

Results of the first splittable quadrupole conduction cooling

Manuscript received July 16, 2013. This work was supported in part by Fermi Research Alliance, LLC, under contract No. DE-AC02-07CH11359 with the U.S. Department of Energy, and in part by the Japan-U.S. cooperative program in High Energy Physics.

M. Tartaglia, R. Carcagno, A. Hemmati, V. Kashikhin, M.J. Kim, L. Kokoska, S. Kotelnikov, J. Nogiec, D. Orris, R. Pilipenko, C. Sylvester, and T. Wokas are with Fermi National Accelerator Laboratory, Batavia, IL 60510 USA (corresponding author phone: 630-840-3890; fax: 630-840-8079; e-mail: tartaglia@fnal.gov).

N. Kimura, H. Hayano, and A. Yamamoto are with the High Energy Accelerator Research Organization (KEK), Tsukuba, Ibaraki, 312-0801 Japan (e-mail: nobuhiro.kimura@kek.jp).

M. Takahashi and T. Tosaka are with Toshiba Corporation in Tokyo, Japan (email: masahico.takahashi@ toshiba.co.jp, taizo.tosaka@ toshiba.co.jp). test, a U.S.-Japan joint R\&D effort, have been recently reported [2,5]. That test was performed at KEK following the quadrupole assembly at the Toshiba Corporation into a new vacuum vessel with conduction cooling channels, and cooled with a $1 \mathrm{~W}$ (at $4 \mathrm{~K}$ ) Sumitomo pulse-tube cryo-cooler. In addition to the test stand design and assembly, Toshiba also machined the quadrupole split yoke faces flat and installed thin soft iron shims to control the surface uniformity and mating of the yoke halves, while maintaining the original geometry. The first test explored the thermal and quench performance of the coils and system; lack of a heater protection system limited testing to low current (up to $30 \mathrm{~A}$ ), and magnetic measurements were not performed at that time.

Subsequently, this test assembly was shipped to Fermilab where it was installed and operated to the full ILC design field, up to $100 \mathrm{~A}$. This paper describes the new test setup, and presents preliminary thermal, quench, and magnetic measurement results from the test. Future plans for the splittable quadrupole test program will be discussed.

\section{Test Setup}

\section{A. Initial Inspection}

The cryostatted magnet assembly mounted in a protective steel frame, cryo-cooler compressor and related components were shipped to Fermilab in October 2012. Shock sensors did not indicate accelerations beyond the limit of $6 \mathrm{~g}$. Once removed from its shipping frame the lateral shipping support was removed and spring tensions for the magnet lateral supports (which accommodate thermal contraction of the supports) were checked. With the bottom superinsulation intact, the bottom vacuum vessel plate was attached and a leak check demonstrated no issues with the vacuum vessel. A check of electrical resistances and hipot of coils and heaters to ground also showed no anomalies.

\section{B. New Test Stand 7}

A new test area was configured for the KEK cryostat assembly in the Fermilab Magnet Test Facility (MTF). Designated "Stand 7", it is located in a previously undeveloped test area of the Industrial Building 1 addition which was originally constructed with a large below-grade pit for a cryogenic test area. This pit is $12 \mathrm{ft}$ by $8 \mathrm{ft}$ wide and $8 \mathrm{ft}$ deep, with electrical services and non-ferromagnetic structures for deck plating at the surface level. A photo of the completed and operating test stand is shown in Fig. 1.

The cryostatted magnet assembly was mounted on a sturdy new aluminum stand anchored in the pit, for convenient access 


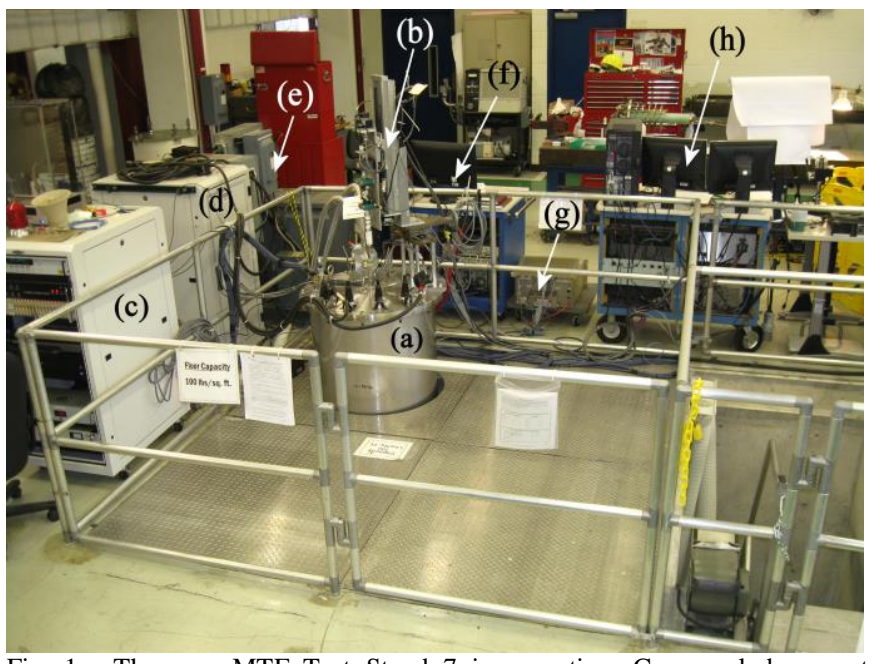

Fig. 1. The new MTF Test Stand 7 in operation. Cryo-cooled magnet assembly (a) with magnetic measurement drive system mounted to top plate (b) is surrounded by test systems: (c) temperature monitoring scan rack, (d) power supply rack and dump cabinet, (e) portable power station, (f) quench detection cart, (g) heater power supply, (h) dsp magnetic measurement cart.

to the top plate. The cryo-cooler compressor and a water chiller to cool it were located down in the pit. With the exception of temperature monitoring data acquisition, the same systems that were previously used to test the splittable quadrupole in MTF Stand 3 [1] (exactly two years earlier) were used: the power supply, dump switch and resistor, quench detection and characterization, heater power supply, and dsp magnetic measurement system are all built into portable racks and carts for deployment where needed. Also the same magnetic measurement drive system, shaft and probe were utilized, with a custom stand-off to mount it above the top plate. The slow scan rack for monitoring temperature and current history was newly assembled, and is based upon a new compactRio architecture controlled by Labview running on a Windows PC, which was recently developed for the Fermilab Solenoid Test Facility [6].

Approval to operate the new Stand 7 was granted on June 17, and the magnet cool-down began on June 18, 2013. The thermal history for the 8 day cool-down from room temperature to $4 \mathrm{~K}$ is shown in Fig. 2, and is in very good agreement with the cool-down in the first test at KEK [2,5]. Initially there was an issue with removing heat from the pit which resulted in the chiller and compressor tripping off due to high temperature. Minor rearrangements of the compressor, chiller, and fans to control the air flow resulted in very stable and reliable operation thereafter. Cold electrical checkout (hipots) passed requirements after cleaning copper lead ceramic insulators.

\section{QUADRUPOLE PERFORMANCE}

The most important goal of the test program was to determine whether stability of the magnetic center was improved by machining of the mating yoke faces, over the full range of operating currents. While conducting the magnetic measurements first, the quench retraining behavior was determined. Additional systematic studies were made to check

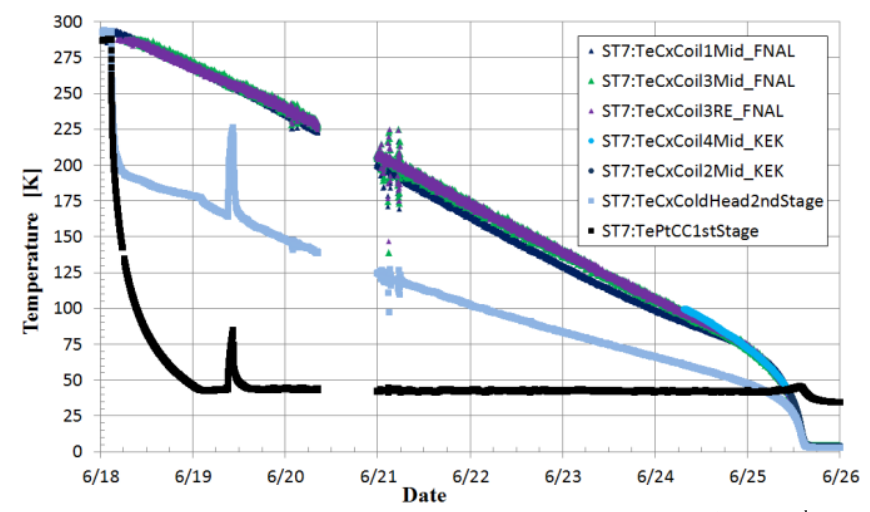

Fig. 2. Cool-down from room temperature to $4 \mathrm{~K}$. Spike in $1^{\text {st }}$ and $2^{\text {nd }}$ stage temperatures corresponds to a 1 hour chiller and compressor trip. Gap in data resulted from monitoring PC accidentally being put to sleep. Noise was caused by welding in a neighboring room.

the conductor critical temperature at and above $30 \mathrm{~A}$, and thermal behavior following a quench: temperature rise and recovery time data are useful, but more important are data attempting to establish whether a quench-back effect (coil heating and quenching due to eddy currents) is present, and whether this may obviate the need for a strip heater protection system. Following each quench (or even firing of the heater), the coil temperatures (measured on the outer surface of the pure aluminum cooling channels) were allowed to return to the initial state values before continuing with another test.

\section{A. Quench Retraining}

The magnetic measurements were taken using "Beam-based alignment" (BBA) profiles, in which the magnet current was ramped to an operating plateau, then ramped down by $20 \%$, and slowly increased in $5 \%$ or $10 \%$ plateau steps back up to the operating point; sometimes with repeated cycles, then ramped down to zero. This process began at a low operating point (10 A), and continued to higher currents: 20, 40, 60, 80, then $100 \mathrm{~A}$.

In the KEK test, no spontaneous re-training quench was seen up to the maximum $30 \mathrm{~A}$ operating current. In the Fermilab Stand 7 test, no re-training quench was seen until the first ramp to $100 \mathrm{~A}$. Only two re-training quenches were recorded, first at $95.9 \mathrm{~A}$ (in coil 1), then at 97.1 A (in coil 4). After these quenches, three stair-step magnetic measurements were performed up to $90 \mathrm{~A}$, then the $100 \mathrm{BBA}$ profile was successfully completed. No spontaneous quenches occurred during the remainder of the test program. Coil 4 had previously shown frequent re-training after thermal cycles, even after having reached $110 \mathrm{~A}$ in the helium bath testing. The short high-current retraining and lack of spontaneous quenches during many low and high current ramps is very encouraging.

\section{B. Magnetic Measurements}

In order to perform measurements using the same tangential coil probe used in [1], the same Stand 3 warm bore tube was used to guide the probe, which has appropriately sized bearings. The KEK cryostat assembly has a built-in warm bore tube that extends the entire length, and the Stand 3 (double walled vacuum insulated) bore tube fits within the aperture. 


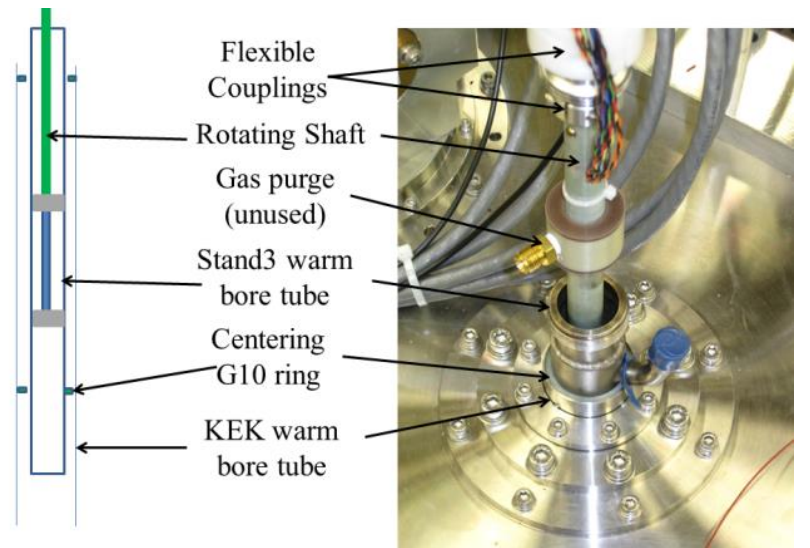

Fig. 3. Nested warm bore tubes and rotating coil shaft. On the left, a schematic section view of the warm bore tube shows bores, rings, and probe.

Because we are interested in measuring center stability at the level of a micron, the challenge is to support one tube securely inside the other. This was done using two G10 centering rings, with an outer diameter matching the KEK tube and an inner diameter matching the Stand 3 tube. Fig. 3 shows a photo of the nested tubes with centering ring, and shaft connecting probe to the drive system.

The G10 rings fit snugly around the MTF tube, but were slightly loose within the KEK bore; however, the tube is not perfectly round along the length, and at several places the G10 rings made a tight fit - especially at the top. One ring was positioned at the top, and another at a location near the bottom of the MTF bore; both rings seemed to fit snugly and no wobble was detectable in the MTF tube.

Initial measurements were taken at $10 \mathrm{~A}$, varying the probe position to find the point at which the $25 \mathrm{~cm}$ long probe was fully into the magnet body. With $\mathrm{Z}=0$ defining the highest probe position, the probe was fully in the body at $Z>11.5 \mathrm{~cm}$. Some measurements were taken at $\mathrm{Z}=12.5 \mathrm{~cm}$, but most were made at $\mathrm{Z}=25.0 \mathrm{~cm}$ near the magnet center. All measurements were taken with a slow $1 / 4 \mathrm{~Hz}$ rotation speed to avoid vibrations. Fig. 4 shows the measured transfer function (gradient/current) for both $\mathrm{Z}$ positions, and from historic data, showing nice agreement.

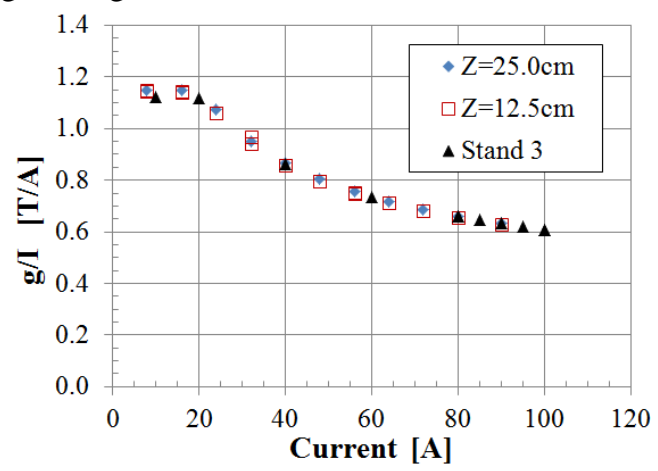

Fig. 4. Normalized quadrupole gradient vs. current for this test and previous measurements.

Previous measurements of the quadrupole harmonics showed that the splittable quadrupole field quality easily met the ILC requirement ( $5 \%$ at $5 \mathrm{~mm}$ radius). Preliminary indications from measurements in this test of the modified quadrupole show that it also easily meets these requirements from low to high field.

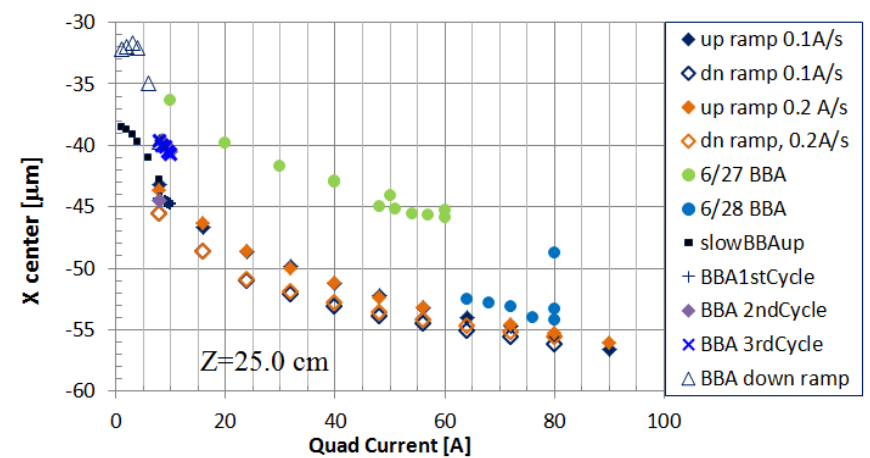

Fig. 5. X center position for many BBA and stairstep measurements.

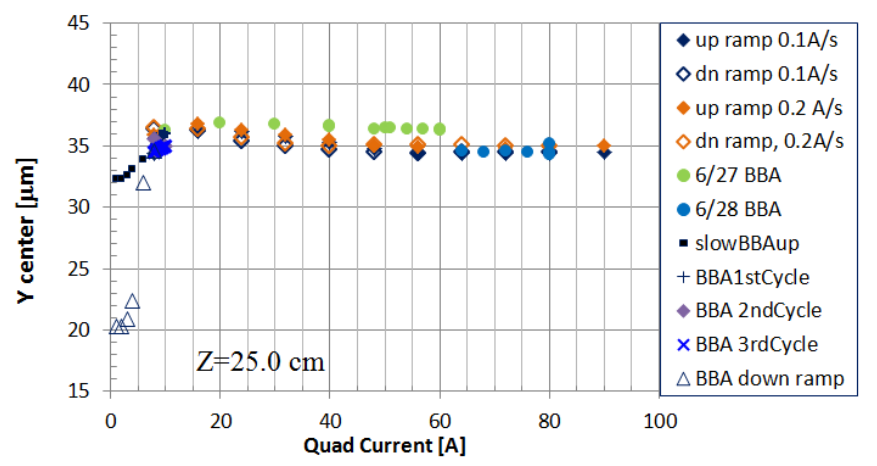

Fig. 6. Y center position for many BBA and stairstep measurements.

Preliminary results on the center position stability can be seen for the $\mathrm{X}$ position (across the yoke gap) in Fig. 5 and $\mathrm{Y}$ position in Fig. 6. In general, the trend is that both are within the requirements, but some unexpected shifts in the positions were seen to occur from one measurement to the next, and within a given measurement. Therefore it is not possible to conclude at this time, and without further study, whether the center stability is adequate.

A number of possibilities could be responsible for these almost random shifts: 1) there may be mechanical instability of the bore or probe bushings; 2) there may be online analysis issues (offline analysis has not yet been completed), 3) there may be unexpected magnetization changes with the coils; 4) local electrical or magnetic changes may influence the measurements (e.g., due to chiller turning off and on). In the near future, these issues will be investigated.

\section{Quench Performance Measurements}

As in the KEK test, critical temperature measurements were taken by powering to fixed current and allowing the temperature to slowly rise after stopping the compressor. Fig. 7 shows the predicted temperatures at the points where data were taken (both KEK and FNAL). However, at Fermilab the measured temperatures are consistently $0.2 \mathrm{~K}$ lower than those predicted and measured at KEK. In fact, the baseline coil temperatures at FNAL are all $0.2 \mathrm{~K}$ below those logged at KEK. Further investigation of this difference, which is large on the scale of the calibration errors $(\sim 0.01 \mathrm{~K})$, will be made. One possibility may be differences in the way calibrations are applied by the instrumentation; another is that FNAL system corrects for thermal emfs by averaging positive and negative sensor excitation, while the KEK system may only use one excitation current polarity. Nevertheless, the agreement is still fairly good, given the limited short sample data and critical surface parametrizations needed to make the comparison. 


\section{Quench Thermal Measurements}

Much data have been collected on the coil and yoke temperature rise and recovery times under a variety of conditions (with and without heater energized, at low and high currents). One basic check to be made is to verify energy balance between heat introduced and temperature rise observed. Also, it will be helpful to further develop an FEA thermal model to better understand thermal resistances and actual coil temperatures, since only the pure aluminum surfaces and magnet bottom temperatures are measured.

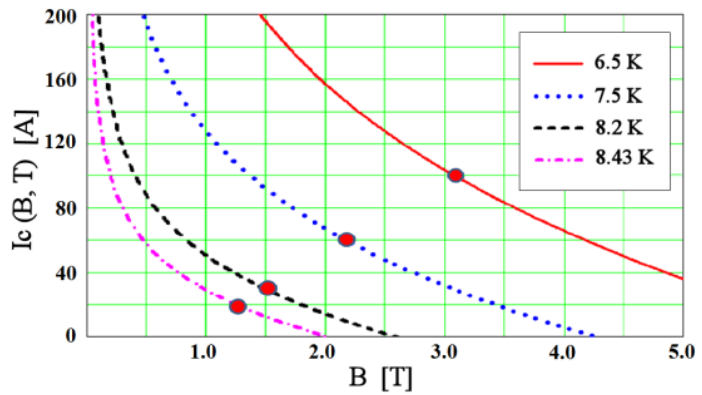

Fig. 7. Predicted field (B) and critical temperature (T) curves with points at crossing of measured quench currents, Ic. Measured quench temperatures at 60 and $100 \mathrm{~A}$ were $0.2 \mathrm{~K}$ below the prediction; the repeated test at $30 \mathrm{~A}$ also was $0.2 \mathrm{~K}$ below. Therefore calibrations are a systematic issue.

In order to evaluate whether quench-back occurs, many data sets have yet to be studied in detail, including low energy quenches with and without heater, as well as spontaneous quenches at high current. Furthermore, comparison of KEK data to FNAL data taken at the same low currents will be very useful. This is because the $\mathrm{dI} / \mathrm{dt}$ profiles are very different following a quench, as shown in Fig. 8, apparently because of very different dump circuit designs: at KEK, relays disconnected the power supply and a $10 \Omega$ dump resistor plus diode were always across the coil. However, at FNAL an IGBT switch opens and the current then redistributes to the $9 \Omega$ resistor. In the latter case, a very fast reduction of the current is seen in the first few milliseconds, so $\mathrm{dI} / \mathrm{dt}$ is very large and this appears to lead to a much faster current decay, suggesting fast resistive voltage growth from quench-back.

\section{Future Plans}

\section{A. Further magnetic measurements}

At this preliminary stage, it is clear that further data analysis is required, as well as investigation of the bore stability and possible external influences on the measurements. It will be possible, certainly at low current (because the HFU may be needed elsewhere), to continue making measurements of center stability. Perhaps the most promising approach would be to utilize a PC-board measurement probe [7] with bearings to match the KEK warm bore tube.

\section{B. Further quench studies}

Thorough analysis of existing data is needed to establish whether quench-back is able to protect the magnet. Once all other measurements are completed, it is conceivable that we may perform a potentially self-destructive test to demonstrate the effect.

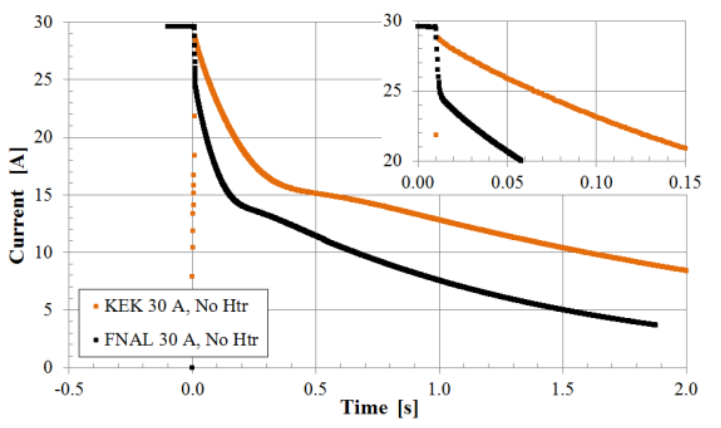

Fig. 8. Current versus time after manual system trips to fire dump at $30 \mathrm{~A}$, with no energizing of the quench heater.

\section{Future conduction cooling tests}

Fermilab is fabricating a short version of the splittable quadrupole, based upon an ASTA (splittable doublet) design. This prototype will be installed into a KEK cryomodule to gain real experience with the installation and operational issues in an accelerating structure. Fabrication of a second identical short magnet is planned, which will be installed into the KEK/Toshiba cryostat where it will be quench tested and have the magnetic field quality and center stability characterized.

\section{CONCLUSION}

A program to test the ILC splittable quadrupole to full current using the KEK/Toshiba cryo-cooled test stand has been carried out, and will continue to explore the magnetic, thermal, and quench performance of this and similar magnets. An important achievement has been the successful exchange and rapid development of the test stand capabilities, which allows comparison of results by very different systems. Studies of the magnetic center position stability and quench performance of the splittable quadrupole have also been extended to the full operating field, and preliminary results are very encouraging but need confirmation.

\section{ACKNOWLEDGMENT}

The authors wish to acknowledge the strong management, technical, and administrative support from the collaborating institutions that made so much possible in such a short time.

\section{REFERENCES}

[1] V. S. Kashikhin, et al., "Superconducting Splittable Quadrupole Magnet for Linear Accelerators," IEEE Transactions on Applied Superconductivity, 22(2012)4002904

[2] N. Andreev, et al., "Conduction Cooling Test of a Splittable Quadrupole for ILC Cryomodules," IEEE Transactions on Applied Superconductivity, 23(2013)3500305.

[3] "International Linear Collider Reference Design Report," http://www.linearcollider.org/cms/?pid=1000025.

[4] http://apc.fnal.gov/programs2/ASTA TEMP/index.shtml

[5] N. Kimura, et al, "Cryogenic Performance of a Conduction Cooling Splittable Quadrupole Magnet for ILC Cryomodules," submitted to the 2013 CEC/ICMC conference.

[6] R. Rabehl, R. Carcagno, J. Nogiec, D. Orris, W. Soyars, C. Sylvester, "A Cryogenic Test Stand for Large Superconducting Solenoid Magnets," submitted to the $2013 \mathrm{CEC} / \mathrm{ICMC}$ conference.

[7] J. DiMarco, et al., "Application of PCB and FDM Technologies to Magnetic Measurement Probe System Development", IEEE Trans. Appl. Supercond. 23(2013)9000505. 